\title{
Probing gluon number density with electron-dijet correlations at EIC
}

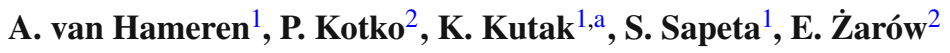 \\ ${ }^{1}$ Institute of Nuclear Physics, Polish Academy of Sciences, Radzikowskiego 152, 31-342 Kraków, Poland \\ ${ }^{2}$ AGH University of Science and Technology, Mickiewicza 30, 30-059 Kraków, Poland
}

Received: 9 July 2021 / Accepted: 6 August 2021 / Published online: 16 August 2021

(C) The Author(s) 2021

\begin{abstract}
We propose a novel way of studying the gluon number density (the so-called Weizsäcker-Williams gluon distribution) using the planned Electron Ion Collider. Namely, with the help of the azimuthal correlations between the total transverse momentum of the dijet system and the scattered electron, we examine an interplay between the effect of the soft gluon emissions (the Sudakov form factor) and the gluon saturation effects. The kinematic cuts are chosen such that the dijet system is produced in the forward direction in the laboratory frame, which provides an upper bound on the probed longitudinal fractions of the hadron momentum carried by scattered gluons. Further cuts enable us to use the factorization formalism that directly involves the unpolarized Weizsäcker-Williams gluon distribution. We find this observable to be very sensitive to the soft gluon emission and moderately sensitive to the gluon saturation.
\end{abstract}

\section{Introduction}

High energy deep inelastic scattering (DIS) of electrons and nuclei at the future electron ion collider (EIC) [1] will provide a unique opportunity to perform detailed studies of various aspects of Quantum Chromodynamics (QCD) [2]. Amongst them is a formation of nuclei in terms of QCD degrees of freedom and, in particular, the dynamics of strongly correlated gluon systems, which leads to emergence of the phenomenon of saturation $[3,4]$.

An especially interesting part of the EIC program concerns jet production [5]. It will allow to study in more detail the internal structure of various hadronic targets, such as $p$, $\mathrm{Pb}, \mathrm{Au}[6,7]$, jet shapes, transport properties of cold nuclear matter [8] low $x$ effects including gluon saturation [9], effects which are not all accessible in inclusive processes. As it has been recognized already in HERA-related studies [10-15]

\footnotetext{
a e-mail: krzysztof.kutak@ifj.edu.pl (corresponding author)
}

(see also [16]), the most suitable processes for the low $x$ studies are those in which jets are produced in the forward direction with respect to the incoming electron, that is they have large rapidities. Kinematically, this allows for a better focus on the events in which the partons extracted from the proton or nucleus carry longitudinal momentum fraction, $x$, which is small enough for the saturation physics to be applicable and tested with high precision.

In the present, work we focus on the production of at least two jets in DIS collisions. Such processes are especially interesting, because, unlike inclusive processes, at high energies, they are directly sensitive to the gluon number density in hadrons, the so-called Weizsäcker-Williams transverse momentum dependent (TMD) gluon distribution $[17,18]$.

The forward dijet production setup can be used reliably only if our theoretical predictions for that process reach certain quality. Specifically, the above kinematic configuration leads to appearance of two groups of potentially large logarithms: $\ln x$ and $\ln \mu$, where the latter, with $\mu$ being of the order of the transverse momenta of the jets, are called the Sudakov logarithms. Both types of logarithms should be resummed simultaneously and such resummation can be performed, as shown in Refs. [19,20], where the results were obtained in position space, using the color dipole formalism [21].

In this work we apply the recently formulated small-x Improved Transverse Momentum Dependent (ITMD) factorization $[22,23]$ which generalizes the small- $x$ TMD factorization [18] to account for power corrections, so that it reduces to the ordinary $k_{T}$ factorization [24] when the saturation effects are neglected. This framework, together with a model for the Sudakov resummation, has recently been successfully applied to shape description of dijet azimuthal angle decorrelations data in $p-p$ and $p-P b$ collisions [25]. ITMD can be regarded as a special case of the Color Glass Condensate (CGC) [26] description once one neglects multiple partonic interactions (the so-called genuine twists) 
[27]. For a detailed study of dijet production at EIC within the full CGC framework see [28].

It is worth mentioning, that higher genuine twits constitute only one of the mechanisms of saturation [29]. The difference between higher genuine twists and the two-body contribution is rather subtle [30,31] and is accessible in rather small transverse momentum domain. It follows, that the higher genuine twists are suppressed at large transverse momenta or photon virtualities, $Q^{2}$. Therefore the ITMD framework is ideally suited for inclusive dijets studies, provided suitable kinematic cuts are imposed. A further advantage of this formalism is that it operates directly in momentum space and therefore allows to relax kinematic approximations often used in the coordinate space calculations. This is especially important for Monte Carlo implementations, which, in particular, allow for a very natural and flexible application of cuts.

In this Letter we propose to utilize the azimuthal correlations between the forward dijet system and the scattered electron at EIC as a tool to study the gluon number distribution. This observable has not been studied in detail in the context of the saturation physics so far. Since there are three tagged final states - the scattered electron and two jets - there is a variety of potentially interesting observables. In studies of the TMD gluon distributions, especially in the saturation regime, the most interesting observables concern azimuthal correlations between the collision products. A quite standard choice is to look at the azimuthal angle between the jets [23,32-35] or hadrons [36,37]. It is obviously the only choice for the dijets at LHC, whereas for the DIS processes, one typically decouples the photon flux from the electron, and thus such observable seems also quite natural. However, in the approaches involving the TMD parton distributions, the transverse momenta of gluons extracted from the proton or nuclei (both internal and due to Sudakov-like soft emissions) are balanced by the whole final state, including the electron. As will be demonstrated in the following sections, the angle between the dijet system and the electron is very sensitive to soft gluon emissions and visibly sensitive to saturation effects Hence, it offers a particularly good handle on quantifying the role Sudakov resummation in dijet production in DIS and its interplay with saturation effects. In general, observables involving scattered electron did not draw a lot of attention in the small- $x$ community so far. Various asymmetries related to the EIC physics have been discussed on general ground in [38]. Quite recently, in [39] the Authors considered azimuthal electron-vector meson (or photon) correlations in exclusive diffractive production within CGC framework.

\section{Framework}

We consider the process of production of a dijet system in DIS

$e+h \rightarrow e^{\prime}+J_{1}+J_{2}+X$,

where $h$ can be a proton or an ion. The corresponding diagram is depicted on the left of Fig. 1, where we also define momenta of the particles involved in the process.

In our calculations, we shall use the ITMD framework, which, as explained in the Introduction, is suitable for our process of interest. It accounts for a complete kinematical twist, which amounts to resumming the $\left(Q_{S} / k_{T}^{2}\right)^{n}$ and $\left(k_{T}^{2} / P_{T}^{2}\right)^{n}$ contributions, where $P_{T} \sim p_{T 1}, p_{T 2}$, which has been shown by a derivation within CGC [27]. Consequently, the offshellness of the initial-state gluon enters both into the TMD gluon density and to the hard matrix element. This way, ITMD generalizes the small- $x$ TMD factorization [18], extending its range of applicability from the case $Q_{s} \ll k_{T} \lesssim$ $p_{T 1}, p_{T 2}$ to $Q_{s} \ll p_{T 1}, p_{T 2}$ and $Q_{s}<k_{T}<p_{T 1}, p_{T 2}$. More precisely, in this work, we employ the ITMD* version of the formalism, with the asterisk indicating that we do not account for linearly polarized gluons in the unpolarized target [40], whose contribution, as discussed below, is suppressed for our choice of kinematical cuts. (See Refs. [38,41-44] for a discussion of linearly polarized gluons in the saturation formalism and beyond.) The ITMD* formula for our process of interest reads

$$
\begin{aligned}
d \sigma_{e h \rightarrow e^{\prime}+2 j+X}= & \int \frac{d x}{x} \frac{d^{2} k_{T}}{\pi} \mathcal{F}_{g g}^{(3)}\left(x, k_{T}, \mu\right) \frac{1}{4 x P_{e} \cdot P_{h}} \\
& \times d \Phi\left(P_{e}, k ; p_{e}, p_{1}, p_{2}\right)\left|\bar{M}_{e g^{*} \rightarrow e^{\prime}+2 j}\right|^{2},
\end{aligned}
$$

where the momenta are denoted such that $P_{h}^{\mu}$ refers to the initial-state hadron, $P_{e}^{\mu}$ to initial-state electron, $k^{\mu}=x P_{h}^{\mu}+$ $k_{T}^{\mu}$ to the initial-state space-like gluon, $p_{e}^{\mu}$ to the final-state electron, and $p_{1,2}^{\mu}$ to the final-state partons, see Fig. 1. The differential phase space element is given by

$$
\begin{gathered}
d \Phi\left(P_{e}, k ; p_{e^{\prime}}, p_{1}, p_{2}\right)=(2 \pi)^{-2} d^{4} p_{e} \delta_{+}\left(p_{e}^{2}\right) d^{4} p_{1} \delta_{+} \\
\left(p_{1}^{2}\right) d^{4} p_{2} \delta_{+}\left(p_{2}^{2}\right) \delta^{4}\left(P_{e}+k-p_{e^{\prime}}-p_{1}-p_{2}\right),
\end{gathered}
$$

so the electrons are assumed to be massless. In the above, the momentum of the off-shell gluon is given by $k^{\mu}=x P_{h}^{\mu}+k_{T}^{\mu}$, with longitudinal momentum fraction $x$ of nucleus $P_{h}^{\mu}$ and transverse momentum such that $P_{h} \cdot k_{T}=0 .|\bar{M}|^{2}$ is the square of the parton-level scattering amplitude summed over spins and colors of the final-state electron and partons and averaged over the initial-state electron spins and gluon colors. Furthermore, it is summed over the allowed flavors of final-state partons. $\mathcal{F}_{g g}^{(3)}\left(x, k_{T}, \mu\right)$ is the hard-scaledependent Weizsäcker-Williams (WW) unpolarized gluon density, counting the number of gluons at resolution scale $\mu$. 
Fig. 1 Dijet production in DIS collision (left), and a graphical representation of the studied observable $\Delta \varphi\left(J_{1}+J_{2}, e^{-}\right)$ (right)
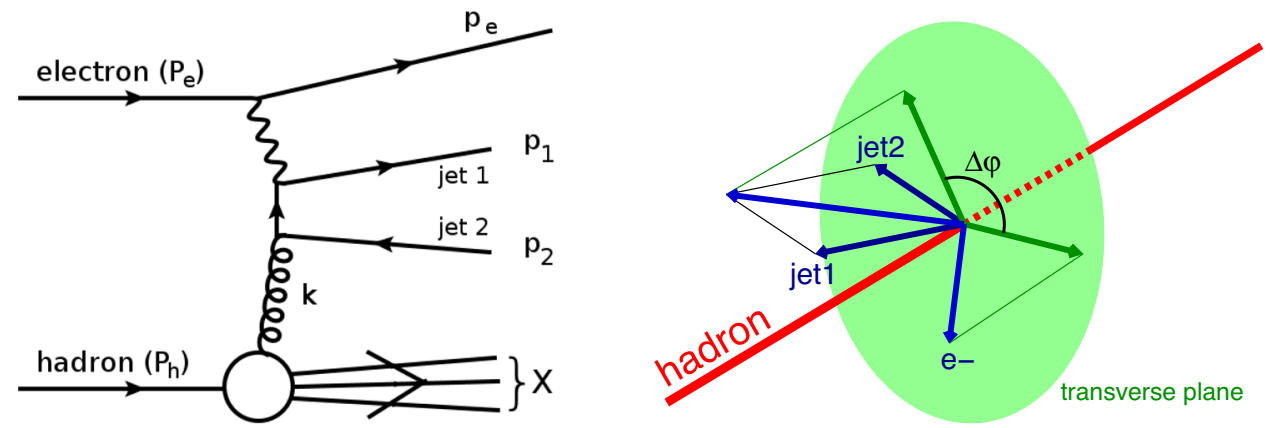

It is given by

$$
\begin{aligned}
\mathcal{F}_{g g}^{(3)}\left(x, k_{T}, \mu\right)= & \int d b_{T} d k_{T}^{\prime} b_{T} k_{T}^{\prime} J_{0}\left(b_{T} k_{T}^{\prime}\right) \\
& \times J_{0}\left(b_{T} k_{T}\right) \mathcal{F}_{g g}^{(3)}\left(x, k_{T}^{\prime}\right) e^{-S_{\text {Sud }}^{g \rightarrow q \bar{q}}\left(\mu, b_{T}\right)},
\end{aligned}
$$

where

$$
\begin{aligned}
\mathcal{F}_{g g}^{(3)}\left(x, k_{T}\right)= & 2 \int \frac{d \xi^{-} d^{2} \xi_{T}}{(2 \pi)^{3} P_{h}^{+}} e^{i x P_{h}^{+}+\xi^{-} i k_{T} \cdot \xi_{T}} \\
& \times\left\langle P_{h}\left|F^{+i}\left(\xi^{-}, \xi_{T}\right) U^{[+] \dagger} F^{+i}(0,0) U^{[+]}\right| P_{h}\right\rangle,
\end{aligned}
$$

is the WW gluon distribution defined in terms of the matrix element of gluon field strength tensor components $F^{+i}$, displaced in the light cone and the transverse directions [45]. $U^{[+]}$are future-pointing gauge links, which make the gluon TMD gauge-invariant. The hard scale dependence of (4) comes from the Sudakov form factor, which itself is a process-dependent object. For dijet production in DIS, the Sudakov at the leading logarithmic approximation and with fixed coupling takes the form [19]

$S_{\mathrm{Sud}}^{g \rightarrow q \bar{q}}\left(\mu, b_{T}\right)=\frac{\alpha_{s} N_{c}}{4 \pi} \ln ^{2} \frac{\mu^{2} b_{T}^{2}}{4 e^{-2 \gamma_{E}}}$,

where, in this work, we take $\alpha_{s}=0.2$.

By using the above gluon density with appropriate evolution (see below) in the cross section formula (2) we achieve simultaneous resummation of small- $x$ and the Sudakov logarithms. The former are relevant because by imposing the appropriate cuts, i.e. selecting the forward jets, we focus the probed longitudinal fractions $x$ on its relatively small values. The latter appear because the production of relatively hard jets introduces additional logarithmic enhancements of the form $\ln p_{T}^{2} / k_{T}^{2}$, which should be resummed. As mentioned above, the factorization formula (2) does not account for the linearly polarized gluons in the unpolarized hadrons. Since we are interested exclusively in the unpolarized WW gluon distributions and the related Sudakov resummation, we shall impose a rather low $Q^{2}$ cut, so that $Q^{2} / P_{T}^{2}$ remains small and thus suppresses the linearly polarized gluon contributions. Even though it is possible to retrieve the linearly polarized part from the CGC formulation (see [31,44]), it is important to be able to test separate components of the calculation.

The WW gluon density can be obtained as a direct solution of the Balitsky-Jalilian-Marian-Iancu-McLerran-WeigertLeonidov-Kovner (B-JIMWLK) evolution equation [4654] or, assuming a Gaussian approximation, it can be constructed from the dipole gluon density obeying the BalitskyKovchegov (BK) equation $[46,55]$. In this paper we choose the latter option since the higher order corrections are better understood for the BK equation and are expected to be more relevant than the simplification due to the Gaussian approximation. Using that, we compute the gluon density from the solution of the BK equation with higher order corrections according to prescription of Kwieciński, Martin and Staśto (KMS) [56,57] yielding the Kutak-Sapeta (KS) gluon density [35] fitted to the proton's $F_{2}$ structure function data. The momentum-space formulation of the BK equation allows us to treat the kinematics exactly. Therefore, the $x$ variable appearing in the formulae above is not the Bjorken $x_{\mathrm{Bj}}=Q^{2} / 2 P_{h} \cdot q$, but the actual fraction of the hadron momentum carried by the scattering gluon, and these two can differ significantly. Within the Gaussian approximation, one derives the following formula for the WW gluon density [23]

$$
\begin{aligned}
\mathcal{F}_{g g}^{(3)}\left(x, k_{T}\right)= & \frac{2 \pi^{2} \alpha_{s}}{N_{c} k_{T}^{2} S_{\perp}} \frac{1}{2} \int_{k_{T}^{2}} d k_{T}^{\prime 2} \ln \frac{k_{T}^{\prime 2}}{k_{T}^{2}} \\
& \times \int \frac{d^{2} q_{T}}{q_{T}^{2}} \mathcal{F}_{q g}^{(1)}\left(x, q_{T}\right) \mathcal{F}_{q g}^{(1)}\left(x, k_{T}^{\prime}-q_{T}\right),
\end{aligned}
$$

where $\mathcal{F}_{q g}^{(1)}$ is the dipole gluon density and $S_{\perp}$ is the target's transverse area.

In Fig. 2 we show the WW KS gluon distributions in proton (left) and lead (right), with and without Sudakov form factors, as functions of the transverse momentum $k_{T}$ and the hard scale $\mu$, for one particular $x=10^{-3}$. (The gluon density is available from the TMDlib [58].) First of all, let us notice that the WW gluon distribution has no maximum, contrary to the dipole gluon $[23,35]$. Secondly, we see that the Sudakov factor suppresses the gluon distribution at low $k_{T}$ and enhances it at higher $k_{T}$. Because the Sudakov form factor is derived in the regime $\mu \propto p_{T} \gg k_{T}$, we apply it 
Fig. 2 The WW gluon density (4), in the proton (left) and lead (right), with and without Sudakov resummation, as a function of the transverse momentum of the gluon, for various values of the hard scale
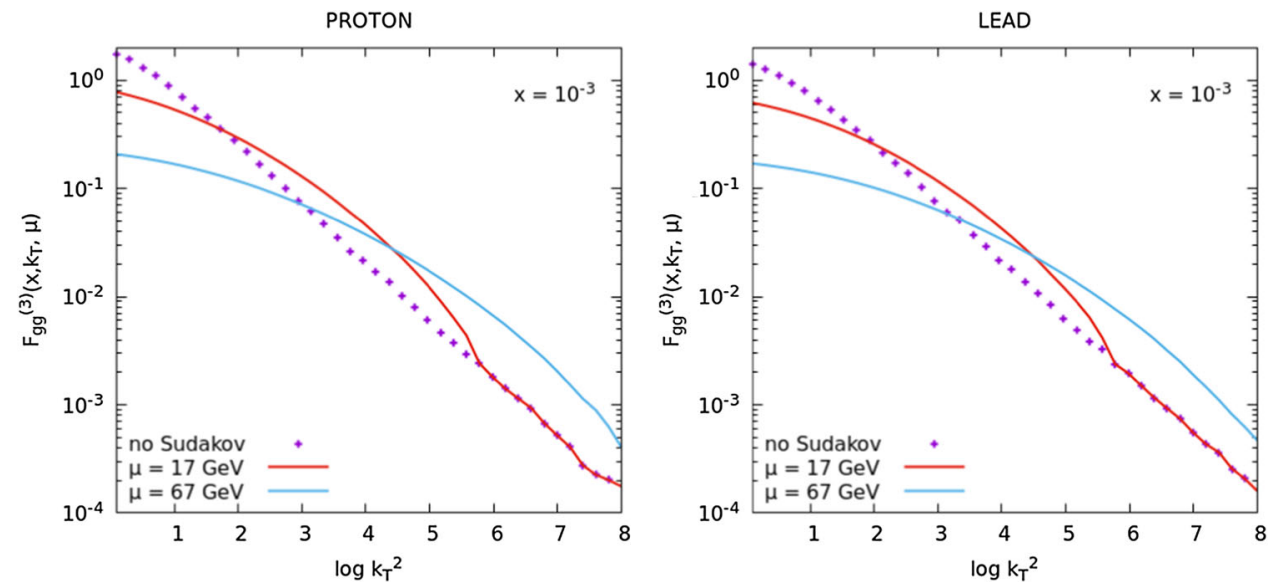
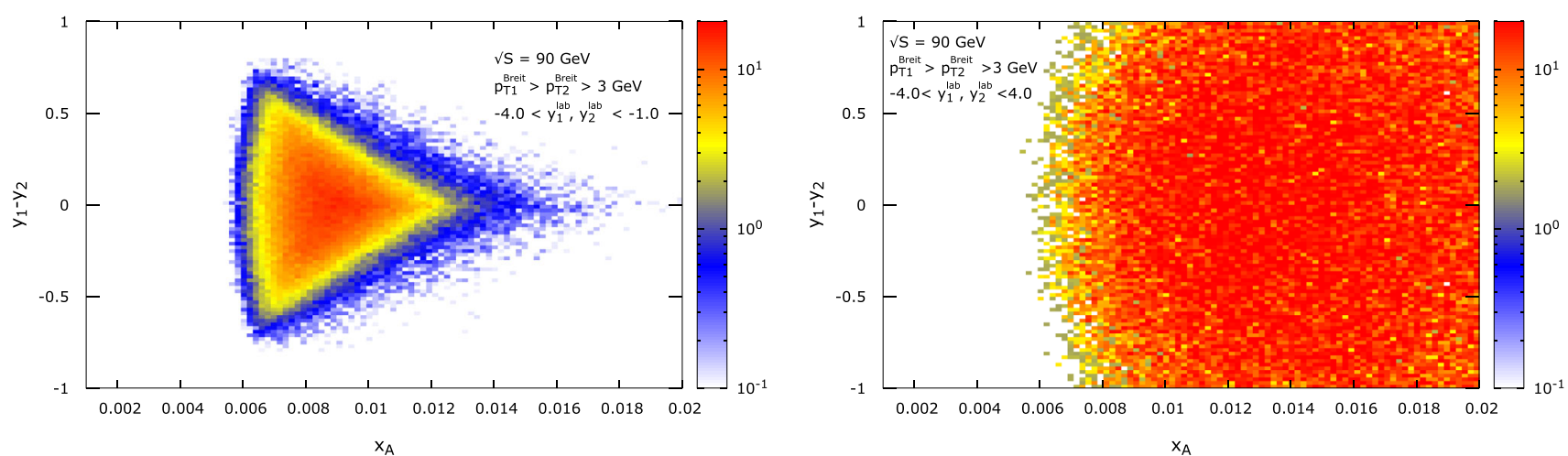

Fig. 3 Density plots representing contribution of events for a given gluon $x$ and jet rapidity difference for the asymmetric rapidity cuts (left) and the symmetric rapidity cuts (right). The asymmetric rapidity cuts guarantee good focusing of the cross section around smaller values of $x$ required by the formalism

only to that part of the gluon density where $\mu>k_{T}$. In the remaining domain, we use the gluon without Sudakov, given in Eq. (7). This is visible in Fig. 2 as a kink of the curve corresponding to $\mu=17 \mathrm{GeV}$. (A similar kink exits also for the $\mu=67 \mathrm{GeV}$ curve but it is located at larger values of $\log k_{T}^{2}$.)

\section{Results}

In this section we present numerical results for the differential cross section as a function of the azimuthal angle between the total transverse momentum of the dijet system and the transverse momentum of the scattered electron, see the right plot of Fig. 1. The calculations are performed both for the $e-p$ and $e-P b$ collisions at the center of mass (CM) energy $\sqrt{S}=90 \mathrm{GeV}$ per nucleon. We look at low-virtuality events, with $Q^{2}>1 \mathrm{GeV}^{2}$, with inelasticity $0.1<v<0.85$. The final-state partons are subject to a jet algorithm, which, at leading order, is simply the cut on the azimuthal anglerapidity plane, i.e. the requirement that $\sqrt{\Delta \Phi^{2}+\Delta y^{2}}<R$. We choose the jet radius $R=1$, as suggested e.g. in Ref.
[5]. The jet definition is imposed in the Breit frame. Furthermore, we require that the transverse momenta of the jets satisfy $p_{T 1}>p_{T 2}>3 \mathrm{GeV}$ in the Breit frame. In order to have a quite narrow distribution of the longitudinal fraction $x$, we select events with the laboratory (LAB) frame rapidities $-4<y_{1}, y_{2}<-1$, where the negative rapidity correspond to the incoming electron beam. The reason for this is illustrated in Fig. 3, where we show density plots for the gluon's $x$ versus the rapidity difference $y_{1}-y_{2}$, for the above asymmetric cut (left) versus the symmetric cut $-4<y_{1}, y_{2}<+4$ (right). We see that the asymmetric cut gives us a very good focusing of the cross section around smaller values of the gluon $x \sim 0.008$. Note, that this corresponds to quite low Bjorken $x_{\mathrm{Bj}} \lesssim 10^{-4}$. On the contrary, the symmetric cut provides a rather broad distribution in the gluon's $x$, extending towards large values, where the formalism is questionable. To summarize, we impose the following cuts:

$$
\begin{aligned}
& \sqrt{S}=90 \mathrm{GeV}, \quad Q^{2}>1 \mathrm{GeV}^{2}, \quad 0.1<v<0.85 \\
& \Delta R^{\text {Breit }}<1, \quad p_{T 1}^{\text {Breit }}>p_{T 2}^{\text {Breit }}>3 \mathrm{GeV}, \\
& -4<y_{1}^{\text {lab }}, y_{2}^{\text {lab }}<-1 .
\end{aligned}
$$




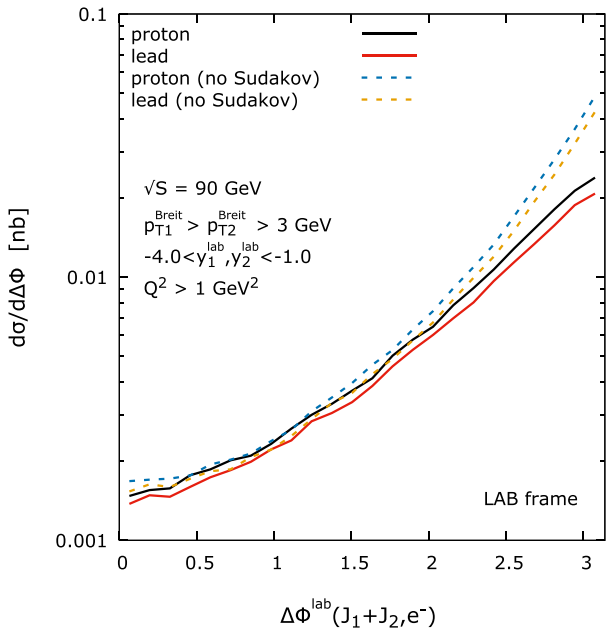

Fig. 4 Azimuthal correlations between the total transverse momentum of the dijet system and the transverse momentum of the scattered electron at EIC in two frames: the LAB frame (left), the Breit frame

We generate events using the KaTie [59] Monte Carlo and present our results both in the laboratory (LAB) frame and the Breit frame. The second option is a preferred choice for dijet observables since it suppresses the LO contributions, i.e. processes dominated by a quark jet [6].

As explained in the previous section, the selection choice (8), with $p_{T}$ 's of jets larger than the $Q_{s}$, is motivated by our goal to study the Sudakov resummation effects together with the kinematical twist [27], in addition to the saturation effects, which, although rather mild, are expected to be present. In other words, the genuine twist configurations (i.e. multiple exchanges of non-soft gluons between the remnant and dijets [29]) as well as contributions from linearly polarized gluons [43] are suppressed. For the latter, we do not impose an upper cut on $Q^{2}$ as the cross section is strongly peaked at low virtualities and such additional cut would introduce only a small correction.

Our predictions for the cross section as a function of the angle between electron and dijet system are presented in Fig. 4. We plot also a control result based on a calculation that neglects the Sudakov form factor. The comparison of the two results clearly shows that while saturation effects are mild, the Sudakov effects are fairly large. This feature is clearly visible in both the $\mathrm{LAB}$ and the Breit frame. In the $\mathrm{LAB}$ frame the difference is concentrated around the correlation region of the dijet-electron system. In the Breit frame, on the other hand, the Sudakov form factor suppresses the cross section over the whole region of the azimuthal angle. Let us note, that the Breit frame is defined in a standard way in our calculation, that is by requiring the photon to have nonzero only the spatial $z$ component. However, unlike in collinear factorization, there is a non-zero transverse momentum of the incoming parton in the factorization formula (2),

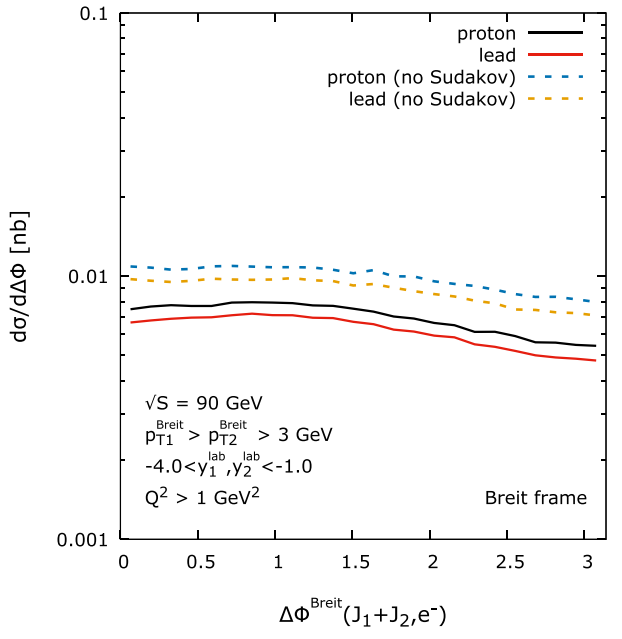

(right). The calculation has been done within the ITMD* framework with the Weizsäcker-Williams gluon distribution obtained from the Kutak-Sapeta fit to HERA data

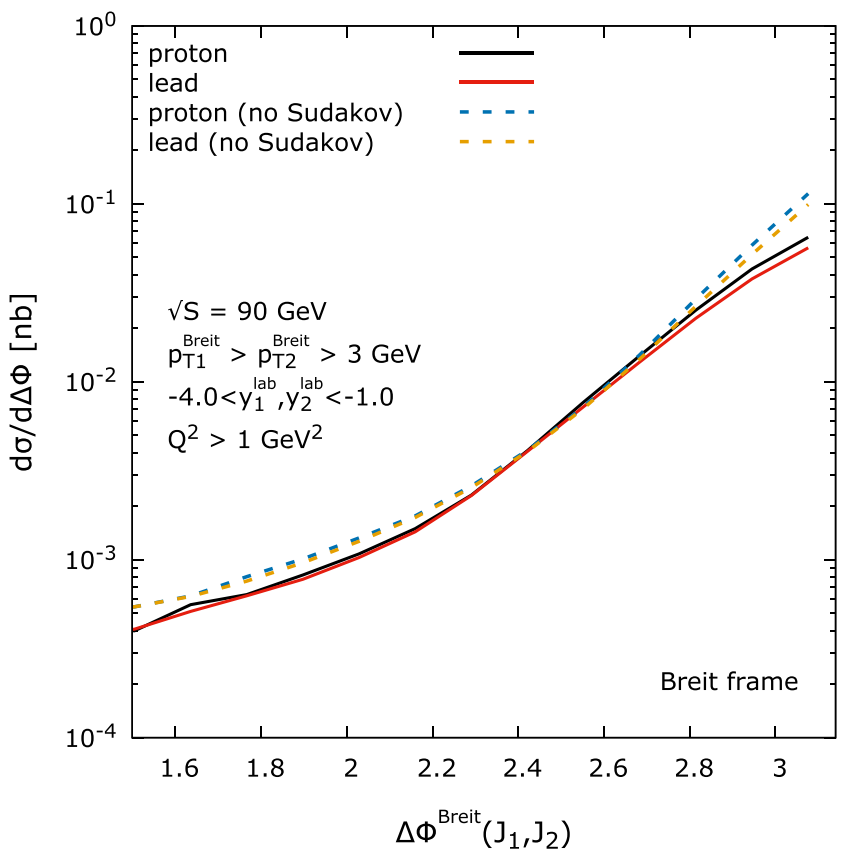

Fig. 5 Azimuthal correlations between the jets in the Breit frame

which makes this frame slightly less intuitive. The saturation effects are observed as an almost constant suppression of the electron-lead cross section (normalized to the number of nucleons) over all azimuthal angles. The suppression reaches maximally about $15 \%$, which although modest, is an observable effect. To be so, the jets must have relatively small $p_{T} \sim 3 \mathrm{GeV}$ in Breit frame, thus, in practice, one can consider dihadron-electron correlations. 


\section{Conclusions}

In this Letter we proposed a new study of the WeizsäckerWilliams gluon distribution, using the azimuthal correlations between the forward dijet system and the scattered electron at the EIC. We chose the kinematic cuts such that both the genuine twists and the linearly polarized gluons are suppressed. This gives us a direct access to the unpolarized distribution via the small- $x$ ITMD factorization. We provided predictions over whole range of the azimuthal angle, which is possible thanks to the inclusion of the kinematic twists and full phase space.

We found that forward dijet-electron azimuthal correlations provide a more sensitive observable to the Sudakov suppression, shown in Fig. 4, as compared to the jet-jet correlations alone, depicted in Fig. 5. In the latter case the difference is concentrated in the region very close to the correlation peak (in Breit frame), whereas for the former observable it extends over large region of azimuthal angle.

Our results may serve as a guidance for future measurements at EIC and improved calculations at the NLO accuracy.

Acknowledgements We acknowledge Hannes Jung for useful correspondence. KK acknowledges informative email exchange with Miguel Arratia. PK is thankful to Farid Salazar for discussions. AvH is supported by grant no. 2019/35/B/ST2/03531 of the Polish National Science Centre. PK is partially supported by National Science Center, Poland, grant no. 2018/31/D/ST2/02731. KK is partially supported by grant no. DEC-2017/27/B/ST2/01985. SS is partially supported by the Polish National Science Centre grant no. 2017/27/B/ST2/02004.

Data Availability Statement This manuscript has no associated data or the data will not be deposited. [Authors' comment: This is a theoretical calculation that is why there is no data associated with it.]

Open Access This article is licensed under a Creative Commons Attribution 4.0 International License, which permits use, sharing, adaptation, distribution and reproduction in any medium or format, as long as you give appropriate credit to the original author(s) and the source, provide a link to the Creative Commons licence, and indicate if changes were made. The images or other third party material in this article are included in the article's Creative Commons licence, unless indicated otherwise in a credit line to the material. If material is not included in the article's Creative Commons licence and your intended use is not permitted by statutory regulation or exceeds the permitted use, you will need to obtain permission directly from the copyright holder. To view a copy of this licence, visit http://creativecomm ons.org/licenses/by/4.0/.

Funded by SCOAP ${ }^{3}$.

\section{References}

1. A. Accardi et al., Electron Ion Collider: The Next QCD Frontier: Understanding the glue that binds us all. Eur. Phys. J. A 52(9), 268 (2016). arXiv:1212.1701

2. R. Abdul Khalek et al., Science requirements and detector concepts for the electron-ion collider: EIC Yellow Report, vol. 3 (2021). arXiv:2103.05419
3. L.V. Gribov, E.M. Levin, M.G. Ryskin, Semihard Processes in QCD. Phys. Rept. 100, 1-150 (1983)

4. A.H. Mueller, J.-W. Qiu, Gluon recombination and shadowing at small values of x. Nucl. Phys. B 268, 427-452 (1986)

5. M. Arratia, Y. Song, F. Ringer, B.V. Jacak, Jets as precision probes in electron-nucleus collisions at the future electron-ion collider. Phys. Rev. C 101(6), 065204 (2020). arXiv:1912.05931

6. X. Liu, F. Ringer, W. Vogelsang, and F. Yuan, Lepton-jet Correlations in Deep Inelastic Scattering at the Electron-Ion Collider. Phys. Rev. Lett. 122 (2019), no. 19, 192003, 1812.08077

7. R. F. del Castillo, M.G. Echevarria, Y. Makris, I. Scimemi, TMD factorization for dijet and heavy-meson pair in DIS. JHEP 01, 088 (2020). arXiv:2008.07531

8. H.T. Li, I. Vitev, Nuclear matter effects on jet production at electron-ion colliders (2020). arXiv:2010.05912

9. I. Kolbé, K. Roy, F. Salazar, B. Schenke, R. Venugopalan, Inclusive prompt photon-jet correlations as a probe of gluon saturation in electron-nucleus scattering at small $x$. JHEP 01, 052 (2021). arXiv:2008.04372

10. A.J. Askew, D. Graudenz, J. Kwiecinski, A.D. Martin, Dijet production at HERA as a probe of BFKL dynamics. Phys. Lett. B 338, 92-97 (1994). arXiv:hep-ph/9407337

11. J. Bartels, V. Del Duca, A. De Roeck, D. Graudenz, M. Wusthoff, Associated jet production at HERA. Phys. Lett. B 384, 300-306 (1996). arXiv:hep-ph/9604272

12. J. Bartels, V. Del Duca, M. Wusthoff, Azimuthal dependence of forward jet production in DIS in the high-energy limit. Z. Phys. C 76, 75-79 (1997). arXiv:hep-ph/9610450

13. J. Kwiecinski, C.A.M. Lewis, A.D. Martin, Deep inelastic events containing two forward jets at HERA. Phys. Rev. D 57, 496-502 (1998). arXiv:hep-ph/9707375

14. J. Kwiecinski, A.D. Martin, A.M. Stasto, Predictions for dijet production in DIS using small x dynamics. Phys. Lett. B 459, 644-648 (1999). arXiv:hep-ph/9904402

15. H1 Collaboration, F. D. Aaron, et al., Measurement of the Azimuthal correlation between the most forward jet and the scattered positron in deep-inelastic scattering at HERA. Eur. Phys. J. C 72, 1910 (2012). arXiv:1111.4227

16. LHeC Study Group Collaboration, J. L. Abelleira Fernandez et al., A Large Hadron Electron Collider at CERN: report on the physics and design concepts for machine and detector. J. Phys. G 39, 075001 (2012). arXiv: 1206.2913

17. D. Kharzeev, Y.V. Kovchegov, K. Tuchin, Cronin effect and high $\mathrm{p}$ (T) suppression in pA collisions. Phys. Rev. D 68, (2003). arXiv:hep-ph/0307037

18. F. Dominguez, C. Marquet, B.-W. Xiao, F. Yuan, Universality of Unintegrated Gluon Distributions at small x. Phys. Rev. D 83, 105005 (2011). arXv:1101.0715

19. A.H. Mueller, B.-W. Xiao, F. Yuan, Sudakov double logarithms resummation in hard processes in the small-x saturation formalism. Phys. Rev. D 88(11), (2013). arXiv:1308.2993

20. A.H. Mueller, B.-W. Xiao, F. Yuan, Sudakov Resummation in Small- $x$ saturation formalism. Phys. Rev. Lett. 110(8), (2013). arXiv: 1210.5792

21. A.H. Mueller, Soft gluons in the infinite momentum wave function and the BFKL pomeron. Nucl. Phys. B 415, 373-385 (1994)

22. P. Kotko, K. Kutak, C. Marquet, E. Petreska, S. Sapeta, A. van Hameren, Improved TMD factorization for forward dijet production in dilute-dense hadronic collisions. JHEP 09, 106 (2015). arXiv: 1503.03421

23. A. van Hameren, P. Kotko, K. Kutak, C. Marquet, E. Petreska, $\mathrm{S}$. Sapeta, Forward di-jet production in $\mathrm{p}+\mathrm{Pb}$ collisions in the small$\mathrm{x}$ improved TMD factorization framework. JHEP 12, 034 (2016). arXiv:1607.03121 
24. S. Catani, M. Ciafaloni, F. Hautmann, High-energy factorization and small $\mathrm{x}$ heavy flavor production. Nucl. Phys. B 366, 135-188 (1991)

25. A. van Hameren, P. Kotko, K. Kutak, S. Sapeta, Broadening and saturation effects in dijet azimuthal correlations in p-p and p- $\mathrm{Pb}$ collisions at $\sqrt{\mathbf{s}}=5.02 \mathrm{TeV}$. Phys. Lett. B 795, 511-515 (2019). arXiv:1903.01361

26. F. Gelis, E. Iancu, J. Jalilian-Marian, R. Venugopalan, The color glass condensate. Ann. Rev. Nucl. Part. Sci. 60, 463-489 (2010). arXiv: 1002.0333

27. T. Altinoluk, R. Boussarie, P. Kotko, Interplay of the CGC and TMD frameworks to all orders in kinematic twist. JHEP 05, 156 (2019). arXiv:1901.01175

28. H. Mäntysaari, N. Mueller, F. Salazar, B. Schenke, Multigluon correlations and evidence of saturation from Dijet measurements at an electron-ion collider. Phys. Rev. Lett. 124(11), 112301 (2020). arXiv: 1912.05586

29. T. Altinoluk, R. Boussarie, Low $x$ physics as an infinite twist (G)TMD framework: unravelling the origins of saturation. JHEP 10, 208 (2019). arXiv: 1902.07930

30. H. Fujii, C. Marquet, K. Watanabe, Comparison of improved TMD and CGC frameworks in forward quark dijet production. JHEP 12, 181 (2020). arXiv:2006.16279

31. R. Boussarie, H. Mäntysaari, F. Salazar, B. Schenke, The importance of kinematic twists and genuine saturation effects in dijet production at the Electron-Ion Collider (2021). arXiv:2106.11301

32. C. Marquet, Forward inclusive dijet production and azimuthal correlations in p(A) collisions. Nucl. Phys. A 796, 41-60 (2007). arXiv:0708.0231

33. M. Deak, F. Hautmann, H. Jung, K. Kutak, Forward Jet Production at the Large Hadron Collider. JHEP 09, 121 (2009). arXiv:0908.0538

34. M. Deak, F. Hautmann, H. Jung, K. Kutak, Forward-central Jet correlations at the large Hadron collider (2010). arXiv:1012.6037

35. K. Kutak, S. Sapeta, Gluon saturation in dijet production in p$\mathrm{Pb}$ collisions at Large Hadron Collider. Phys. Rev. D 86, (2012). arXiv: 1205.5035

36. J.L. Albacete, C. Marquet, Azimuthal correlations of forward dihadrons in $\mathrm{d}+\mathrm{Au}$ collisions at RHIC in the Color Glass Condensate. Phys. Rev. Lett. 105, (2010). arXiv:1005.4065

37. L. Zheng, E.C. Aschenauer, J.H. Lee, B.-W. Xiao, Probing Gluon Saturation through Dihadron Correlations at an Electron-Ion Collider. Phys. Rev. D 89(7), (2014). arXiv:1403.2413

38. D. Boer, P.J. Mulders, C. Pisano, J. Zhou, Asymmetries in Heavy Quark Pair and Dijet Production at an EIC. JHEP 08, 001 (2016). arXiv: 1605.07934

39. H. Mäntysaari, K. Roy, F. Salazar, B. Schenke, Gluon imaging using azimuthal correlations in diffractive scattering at the Electron-Ion Collider. Phys. Rev. D 103(9), 094026 (2021). arXiv:2011.02464

40. P.J. Mulders, J. Rodrigues, Transverse momentum dependence in gluon distribution and fragmentation functions. Phys. Rev. D 63, (2001). arXiv:hep-ph/0009343

41. A. Metz, J. Zhou, Distribution of linearly polarized gluons inside a large nucleus. Phys. Rev. D 84, (2011). arXiv:1105.1991
42. F. Dominguez, J.-W. Qiu, B.-W. Xiao, F. Yuan, On the linearly polarized gluon distributions in the color dipole model. Phys. Rev. D 85, (2012). arXiv: 1109.6293

43. C. Marquet, C. Roiesnel, and P. Taels, Linearly polarized small- $x$ gluons in forward heavy-quark pair production. Phys. Rev. D 97(1), 014004 (2018). arXiv:1710.05698

44. T. Altinoluk, C. Marquet, P. Taels, Low-x improved TMD approach to the lepto- and hadroproduction of a heavy-quark pair. JHEP 06, 085 (2021). arXiv:2103.14495

45. F. Dominguez, B.-W. Xiao, F. Yuan, $k_{t}$-factorization for hard processes in nuclei. Phys. Rev. Lett. 106, (2011). arXiv:1009.2141

46. I. Balitsky, Operator expansion for high-energy scattering. Nucl. Phys. B 463, 99-160 (1996). arXiv:hep-ph/9509348

47. J. Jalilian-Marian, A. Kovner, A. Leonidov, H. Weigert, The BFKL equation from the Wilson renormalization group. Nucl. Phys. B 504, 415-431 (1997). arXiv:hep-ph/9701284

48. J. Jalilian-Marian, A. Kovner, A. Leonidov, H. Weigert, The Wilson renormalization group for low x physics: towards the high density regime. Phys. Rev. D 59, (1998). arXiv:hep-ph/9706377

49. J. Jalilian-Marian, A. Kovner, H. Weigert, The Wilson renormalization group for low x physics: Gluon evolution at finite parton density. Phys. Rev. D 59, (1998). arXiv:hep-ph/9709432

50. A. Kovner, J.G. Milhano, H. Weigert, Relating different approaches to nonlinear QCD evolution at finite gluon density. Phys. Rev. D 62, (2000). arXiv:hep-ph/0004014

51. A. Kovner, J.G. Milhano, Vector potential versus color charge density in low $x$ evolution. Phys. Rev. D 61, (2000). arXiv:hep-ph/9904420

52. H. Weigert, Unitarity at small Bjorken x. Nucl. Phys. A 703, $823-$ 860 (2002). arXiv:hep-ph/0004044

53. E. Iancu, A. Leonidov, L.D. McLerran, Nonlinear gluon evolution in the color glass condensate. 1. Nucl. Phys. A 692, 583-645 (2001). arXiv:hep-ph/0011241

54. E. Ferreiro, E. Iancu, A. Leonidov, L. McLerran, Nonlinear gluon evolution in the color glass condensate. 2. Nucl. Phys. A 703, 489538 (2002). arXiv:hep-ph/0109115

55. Y.V. Kovchegov, Small x F(2) structure function of a nucleus including multiple pomeron exchanges. Phys. Rev. D 60, (1999). arXiv:hep-ph/9901281

56. J. Kwiecinski, A.D. Martin, A.M. Stasto, A Unified BFKL and GLAP description of F2 data. Phys. Rev. D 56, 3991-4006 (1997). arXiv:hep-ph/9703445

57. K. Kutak, J. Kwiecinski, Screening effects in the ultrahighenergy neutrino interactions. Eur. Phys. J. C 29, 521 (2003). arXiv:hep-ph/0303209

58. N.A. Abdulov et al., TMDlib2 and TMDplotter: a platform for 3D hadron structure studies (2021). arXiv:2103.09741

59. A. van Hameren, KaTie: For parton-level event generation with $k_{T}$-dependent initial states. Comput. Phys. Commun. 224, 371-380 (2018). arXiv: 1611.00680 